\title{
ROC curves for recognition of visual patterns'
}

\author{
Wayne Lee \\ UNIVERSITY OF CALIFORNIA, BERKELEY
}

\begin{abstract}
The confidence rating technique was used to generate $\mathrm{z}$-deviate ROC curves for the recognition of one of two possible visual patterns. The patterns were O's and Landolt rings. The mean linear slopes for each pattern pair were about $45^{\circ}$, but there was some evidence of downward curvilinearity.
\end{abstract}

\section{Problem}

The basic ideas of statistical decision theory have been widely applied in psychophysics under the name "detection theory." In an explanation and review of this area, Swets, Tanner, \& Birdsall (1961) have proposed that the theory is applicable to stimuli of any degree of complexity, and that it provides insight into perceptual processes in general, not just into the sensory processes of psychophysics.

The purpose of the present study was to apply the techniques of statistical detection theory to the recognition of visual patterns under conditions of difficult discriminability. Receiver-operating-characteristic (ROC) curves were derived from the data and examined to see whether they were consistent with normaldistribution theory.

\section{Method}

Two sets of three stimuli each were used (Fig. 1). During a series of trials, an S was presented with one stimulus from a pair from one set. At different times, an $\mathrm{S}$ was presented all three pairs possible from a set of three stimuli. Five Ss were used with the first set, and five with the second. Subjects were told which pair of patterns the stimuli were taken from for each series of trials. Subjects were John Hopkins University undergraduate males who participated in order to fulfill a class requirement.

Each $\mathrm{S}$ was run individually for one session a week over a period of several weeks. During each session, warmup trials were given, and then all the different series (pairs) were presented in different orders. For each trial, one of the pair of stimuli was randomly selected, and flashed tachistoscopically on a white cardboard screen. Then $\mathrm{S}$ wrote his opinion as to which of the pair was flashed, and gave a confidence rating. No feedback was given. The confidence categories were: (1) pure guess, (2) may be, (3) good chance, (4) fairly certain, (5) positive. The presentation time and contrast were adjusted individually by trial and error to produce a moderate amount of confusion. Since $\mathrm{S}$ improved during each session with warmup, conditions were made continuously harder during warmup until it seemed that $S$ had reached a plateau.
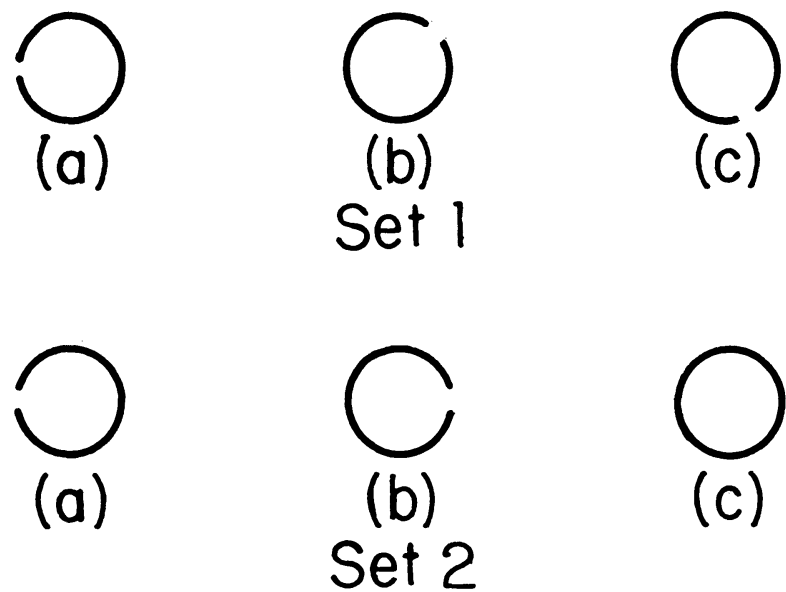

Fig. 1. The two sets of patterns used as stimuli.

\section{Results}

Subjects were able to give valid confidence ratings. The percentage of correct responses for the five confidence categories, averaged over all Ss, were: (1) 60, (2) 70, (3) 84, (4) 92 , and (5) 96.

ROC curves were plotted for each $\mathrm{S}$ for each of the three pairs in the set. A best linear fit was plotted by eye, and the angles measured in degrees. Equalvariance normal distribution detection theory predicts that this line will have a slope of $45^{\circ}$. One would think that Pair $a-b$ and Set 2 would have the best chance to meet the equal-variance condition, because of figure symmetry. Likewise, all pairs from Set 1 have something of the same symmetric quality. Pairs $a-c$ and $b-c$ from Set 2 might be expected to not have equal variance, however. Actually, the mean slopes for all pairs (averaged across five Ss) were very close to $45^{\circ}$. The largest mean deviation was for Pair $a-b$ of Set 2, for which the mean slope was $48.6^{\circ}$. None of the mean slopes even approached significance when tested for deviation from $45^{\circ}$. Thus, although the slopes of individual Ss varied widely, it would appear that all pairs used in this experiment, when averaged across Ss, have equal internal dispersion.

In order to further verify the theory, it must be shown that the lines are straight-without curvature. In order to observe curvature, the extreme points of each ROC curve were omitted, and a best straight line was applied to the remaining points. Then if the majority of the omitted points were below this fitted line, the curve was said to have a downward curvature. 
If the majority of the omitted points were above, the curve was said to have an upward curvature. In some cases, there was no preponderance. A chi-square test on only those 21 of the 30 curves which had a curvature up or down yielded a significance level of .05. However, if those curves without up or down curvature were divided between the two curvature groups equally, then the significance level was only .10. (The data were examined and found to be uncorrelated for curvature within Ss; therefore all 30 curves were treated as independent data for the chi-square test, although there were actually three curves for each of $10 \mathrm{Ss}$.)

\section{Diseussion}

In general, the confidence rating technique for deriving ROC curves proved to be efficient for use with tachistoscopic presentation of visual patterns. For the Landolt ring patterns used here, all patterns appear to be equally confusable internally, when averaged over Ss. There is a suggestion that the normal distribution theory is somewhat in error, however, since it predicts a straight line for the $\mathrm{z}$-deviate ROC curves, but there is a strong suggestion of average downward curvature in the data. There are many possible explanations for this. The explanation might be in a failure of the strict decision theory cutoff mode of responding, although Lee (1963) found that wide deviations from the cutoff mode of responding could still result in straight ROC curves. The discriminability might have varied somewhat from session to session, against the theory assumptions; but when theoretical curves were derived based on combining data from two discriminability levels, the ROC curves were actually curved upward slightly. Perhaps the best guess is that for patterns, the internal distributions postulated do not have the simple normal form.

\section{References}

LEE, W. Choosing among confusably distributed stimuli with specified likelihood ratios. Percept. mot. Skills, 1963, 16, 445-467.

SWETS, J. A., TANNER, W. P., JR., \& BIRDSALL, T. G. Decision processes in perception. Psychol. Rev., 1961, 68, 301-340. Note

1. This research was supported in part by NIH grant GM 11128 . 